\title{
Present Status of Theory and Experiment as to Atomic Disintegration and Atomic Synthesis.*
}

\author{
By Prof. Robert A. Millikan, California Institute of Technology, Pasadena, California.
}

$\mathrm{M}^{\mathrm{Y}}$ $Y$ task is to attempt to trace the history of the development of scientific evidence bearing on the question of the origin and destiny of the physical elements. I shall list ten discoveries or developments, all made within the past hundred years, which touch in one way or another upon this problem and constitute indications or sign-posts on the road toward an answer.

Prior to the middle of the nineteenth century, little experimental evidence of any sort had appeared, so that the problem was wholly in the hands of the philosopher and the theologian. Then came, first, the discovery of the equivalence of heat and work, and the consequent formulation of the principle of the conservation of energy, probably the most farreaching physical principle ever developed.

Following this, and directly dependent upon it, came, second, the discovery, or formulation, of the second law of thermodynamics, which was first interpreted, and is still interpreted by some, as necessitating the ultimate 'heat-death' of the universe and the final extinction of activity of all sorts ; for all hot bodies are observed to be radiating away their heat, and this heat after having been so radiated away into space apparently cannot be reclaimed by man. This is classically and simply stated in the humpty-dumpty rhyme. As a natural if not necessary corollary to this was put forward by some, in entire accord with the demands of medieval theology, a Deus ex machina initially to wind up or start off this running-down universe.

Then came, third, the discovery, through studies both in geology and biology, of the facts of evolution-facts which showed that, so far as the biological field is concerned, the process of creation, or upbringing from lower to higher forms, has been continuously going on for millions upon millions of years and is presumably going on now. This tended to direct attention away from the Deus ex machina, to identify the Creator with his universe, to strengthen the theological doctrine of immanence, which represents substantially the philosophic position of Leonardo da Vinci, Galileo, Newton, Francis Bacon, and most of the great minds of history down to Einstein.

Neither evolution nor evolutionists have in general been atheistic-Darwin least of all-but their influence has undoubtedly been to raise doubts about the legitimacy of the dogma of the Deus ex machina and of the correlative one of the heat-death. This last dogma rests squarely on the assumption that we, infinitesimal mites on a speck of a world, know all about how the universe behaves in all its parts, or more specifically, that the radiation laws which seem to us to hold here cannot possibly have any exceptions anywhere, even though that is precisely the sort of sweeping generalisation that has led us physicists into error half a dozen times

- Retiring presidential address to the American Association for the Advancement of Science, delivered at Cleveland on Dec. 29.

No. 3196, VoL. 127] during the past thirty years, and also though we know quite well that conditions prevail outside our planet which we cannot here duplicate or even approach. Therefore the heat-death dogma has always been treated with reserve by the most thoughtful of scientific workers. No more crisp or more cogent statement of what seems to me to be the correct position of science in this regard has come to my attention than is found in the following recent utterance of Gilbert $\mathrm{N}$. Lewis, namely, "Thermodynamics gives no support to the assumption that the universe is running down". "Gain of entropy always means loss of information and nothing more."

The fourth discovery bearing on our theme was the discovery that the dogma of the immutable elements was definitely wrong. By the year 1900 the element radium had been isolated and the mean lifetime of its atoms found to be about two thousand years. This meant definitely that the radium atoms that are here now have been formed within about that time; and a year or two later the element helium was definitely observed to be growing out of radium here and now. This raised insistently the question as to whether the creation, or at least the formation, of all the elements out of something else may not be a continuous processstupendous change in viewpoint the discovery of radioactivity brought about, and a wholesome lesson of modesty it taught to the physicist. But a couple of years later, uranium and thorium, the heaviest known elements, were definitely caught in the act of begetting radium, and all the allied chain of disintegration products. Since, however, the lifetime of the parent atom, uranium, has now been found to be a billion years or so, we have apparently ceased to inquire whence it comes. We are disposed to assume, however, that it is not now being formed on earth. Indeed, we have good reason to believe that the whole radioactive process is confined to a very few, very heavy elements which are now giving up the energy which was once stored up in them-we know not how-so that radioactivity, though it seemed at first to be pointing away from the heat-death, has not at all, in the end, done so. Indeed, it seems to be merely one mechanism by which stored-up energy is being frittered away into apparently unreclaimable radiant heat-another case of humpty-dumpty.

The fifth significant discovery was the enormous lifetime of the earth-partly through radioactivity itself, which assigns at least a billion and a half years - and the still greater lifetime of the sun and stars-thousands of times longer than the periods through which they could possibly exist as suns if they were simply hot bodies cooling off. This meant that new and heretofore unknown sources of heat energy had to be found to keep the stars pouring out such enormous quantities of radiation for such ages upon ages. 
The sixth discovery, and in many ways the most important of all, was the development of evidence for the interconvertibility of mass and energy. This came about in three ways. In 1901 Kaufman showed experimentally that the mass of an electron could be increased by increasing sufficiently its velocity: that is, energy could be definitely converted into mass. About the same time the pressure of radiation was experimentally established by Nichols and Hull at Dartmouth College, New Hampshire, and Lebedew at Moscow. This meant that radiation possesses the only distinguishing property of mass, the property by which we define it, namely, inertia. The fundamental distinction between radiation and matter thus disappeared. These were direct, experimental discoveries. Next, in 1905, Einstein developed the interconvertibility of mass and energy as a necessary consequence of the special theory of relativity. If, then, the mass of the sun could in any way be converted into radiant heat, there would be an abundant source of energy to keep the sun going so long as necessary, and all our difficulties about the lifetimes of the sun and stars would have disappeared. But what could be the mechanism of this transformation?

Then came the seventh discovery, which constituted a very clear finger-post, pointing to the possibility of the existence of an integrating or building-up process among the physical elements, as well as in biological forms, in the discovery that the elements are all definitely built up out of hydrogen; for they-the ninety-two different atoms-were all found, beginning about 1913 by the new method of so-called positive ray analysis, to be exact multiples of the weight of hydrogen within very small limits of uncertainty. This fact alone raises very insistently the query as to whether they are not being built up somewhere out of hydrogen now. They certainly were once so put together, and some of them, the radioactive ones, are now actually caught in the act of splitting up. Is it not highly probable, so would say any observer, that the inverse process is going on somewhere, especially since the process would involve no violation either of the energy principle or of the second. law of thermodynamies; for hydrogen, the element out of which they all must be built, has not a weight exactly one in terms of the other ninety-two, but about 1 per cent more than one, so that since mass or weight had been found in the sixth discovery to be expressible in terms of energy, the union of any number of hydrogen atoms into any heavier element, meant that 1 per cent of the total available potential energy had disappeared and was therefore available for appearance as heat.

When, about 1914-15, this fact was fitted by MacMillan, Harkins, and others into the demand made above in the fifth discovery for a new source of energy to keep the sun pouring out heat so copiously for such great lengths of time, it seemed to the whole world of physics that the building up of the heavier elements out of hydrogen under the conditions existing within the sun and stars had been practically definitely proved to be taking place. This would not provide an escape from the heat-death, but it would enormously postpone it, that is, until all the hydrogen in the universe had. been converted into the heavier elements.

By this process, however, the suns could stoke at most but 1 per cent of their total mass, assuming they were wholly hydrogen to begin with, into their furnaces, and 99 per cent of the mass of the universe would remain as cold, dead ash when the fires were all gone out and the heat-death had come. But about 1917 the astronomer began to chafe under the time-limitation thus imposed upon him, and this introduced the eighth consideration bearing upon our theme. He could get a hundred times more time-from now on, much more than that, because only a small fraction of the matter in the universe is presumably now hydrogen-by assuming that, in the interior of heavy atoms, occasionally a negative electron gets tired of life at the pace it has to be lived in the electron world, and decides to end it all and commit suicide ; but, being paired by Nature in electron-fate with a positive, he has to arrange a suicide pact with his mate, and so the two jump into each other's arms in the nucleus, and the two complementary electron lives are snuffed out at once; but not without the letting loose of a terrific death-yell, for the total mass of the two must be transformed into a powerful ether pulse which, by being absorbed in the surrounding matter, is supposed to keep up the mad, hot pace in the interiors of the suns. This discovery, or suggestion, to account for the huge estimated stellar lifetimes, of the complete annihilation of positive and negative electrons within the nucleus, makes it unnecessary to assume, at least for stellar lifetime purposes, the building up of the heavier elements out of hydrogen. Indeed, it seems rather unlikely that both kinds of processes, atom-building and atomannihilating, are going on together in the same spot under the same conditions; so we must turn to further experimental facts to get more light.

The ninth sign-post came into sight in 1927, when Aston made a most precise series of measurements on the relative masses of the atoms, which made it possible to subject to a new test the Einstein formula for the relation between mass and energy, namely, $E=M c^{2}$. This Aston curve is one of the most illuminating finger-pointings we now have. It shows that:

1. Einstein's equation actually stands the quantitative test for radioactive or disintegrating processes right well, and therefore receives new experimental credentials.

2. The radioactive or disintegrating process with the emission of an alpha ray must be confined to a very few heavy elements, since these are the only ones so situated on the curve that mass can disappear, and hence heat energy appear, through such disintegration.

3. All the most common elements, except hydrogen, are already in their most stable condition, that is, their condition of minimum mass, so that if we disintegrate them we shall have to do work upon them, rather than get energy out of them.

4. Therefore, man's only possible source of

$$
\text { No. 3196, VoL. 127] }
$$


energy other than the sun is the upbuilding of the common elements out of hydrogen or helium, or else the entire annihilation of positive and negative electrons; and there is no likelihood that either of these processes is a possibility on earth.

5. If the foregoing upbuilding process is going on anywhere, the least penetrating and the most abundant radiation produced by it, that corresponding to the formation of helium out of hydro. gen, ought to be about ten times as energetic as the hardest gamma rays, that is, it ought to correspond to about twenty-six million electron-volts in place of two and a half million.

6. Other radiations corresponding to the only other abundant elements, namely, oxygen (oxygen, nitrogen, carbon), silicon (magnesium, aluminium, silicon), and iron (iron group), should be found about four times, seven times, and fourteen times as energetic as the 'helium rays'.

7. The radiation corresponding to the smallest annihilation process that can take place-the suicide of a positive and negative electron-is three hundred and fifty times as energetic as the hardest gamma ray, or thirty-five times as energetic as the 'helium ray'.

This brings us to the tenth discovery, that of the cosmic rays. These reveal :*

1. A radiation, the chief component of which, according to our direct comparison, is five times as penetrating as the hardest gamma ray, which, with the best theoretical formula we have relating energy and penetrating power (Klein-Nishina), means a ray ten times as energetic as the hardest gamma ray, precisely according to prediction.

2. Special bands of cosmic radiation that are roughly where they should be to be due to the formation of the foregoing abundant elements out of hydrogen, though (for reasons to be given presently) no precise quantitative check is to expected except in the case of helium.

3. No radiation of significant amount anywhere near where it is to be expected from the annihilation hypothesis, thus indicating that at least 95 per cent of the observed cosmic rays are due to some other less energetic processes.

4. A radiation that is completely independent of the sun, the great hot mass just off our bows, and not appreciably dependent on the Milky Way or the nearest spiral nebula, Andromeda, one that comes in to us practically uniformly from all portions of the celestial dome, and is so invariable with both time and latitude at a given elevation that the observed small fluctuations at a given station reflect with much fidelity merely the changes in the thickness of the absorbing air blanket through which the rays have had to pass to get to the observer.

This last property is the most amazing and the most significant property exhibited by the cosmic rays, and before drawing the final conclusions its significance will be discussed. For it means that at the time these rays enter the earth's atmosphere, they are practically pure ether waves or photons.

* See articles by Millikan and by Millikan and Cameron, Phys. Rev. Dee. 1,1930 , and in press.

No. 3196 , Vor. 127]
If they were high-speed electrons or even had been appreciably transformed by Compton encounters in passing through matter into such high-speed electrons or beta rays, these electrons would of necessity spiral about the lines of force of the earth's magnetic field and thus enter the earth more abundantly near the earth's magnetic poles than in lower latitudes. This is precisely what the experiments made during the last summer at Churchill, Manitoba (lat. $59^{\circ}$ N.), within 730 miles of the north magnetic pole, showed to be not true, the mean intensity of the rays there being not measurably different from that at Pasadena in lat. $34^{\circ} \mathrm{N}$.

Nor is the conclusion that the cosmic rays enter the earth's atmosphere as a practically pure photon beam dependent upon these measurements of last summer alone. It follows also from the high altitude sounding-balloon experiments of Millikan and Bowen in April 1922, taken in connexion with the lower balloon flights of Hess and Kolhörster in 1911-14. For in going to an altitude of $15.5 \mathrm{~km}$. we got but one-fourth the total discharge of our electroscope which we computed we should have obtained from the extrapolation of our predecessors' curves. This shows that somewhere in the atmosphere below a height of $15.5 \mathrm{~km}$. the intensity of the ionisation within a closed ressel exposed to the rays goes through a maximum, and then decreases, quite rapidly, too, in going to greater heights. We have just taken very accurate observations up to the elevation of the top of Pike's Peak $(4.3 \mathrm{~km}$.), and found that within this range the rate of increase with altitude is quite as large as that found in the Hess and Kolhörster balloon flights, so that there can be no uncertainty at all about the existence of this maximum. Such a maximum, however, means that the rays, before entering the atmosphere, have not passed through enough matter to begin to get into equilibrium with their secondaries-beta rays and photons of reduced frequency - in other words, that they have not come through an appreciable amount of matter in getting from their place of origin to the earth.

This checks with the lack of effect of the earth's magnetic field on the intensity of the rays; and the two phenomena, of quite unrelated kinds and brought to light years apart, when taken together, prove most conclusively, I think, that the cosmic rays cannot originate even in the outer atmospheres of the stars, though these are full of hydrogen and helium in a high temperature state, but that they must originate rather in those portions of the universe from which they can come to the earth without traversing matter in quantity that is appreciable even as compared with the thickness of the earth's atmosphere-in other words, that they must originate in the intensely cold regions in the depths of interstellar space.

Further, the more penetrating the beta rays produced by Compton encounters, the greater the thickness of matter that must be traversed before the beam of pure photons which enters the atmosphere gets into equilibrium with its secondaries: and until such equilibrium is reached, the apparent 
absorption coefficient must be less than the coefficient computed with the aid of the KleinNishina formula from the energy released in the process from which the radiation arises. Now the Bothe-Kolhörster experiments of about a year ago show that when the energies of the incident photons are sufficiently high, the beta rays released by Compton encounters do indeed become abnormally penetrating: so that it is to be expected that, for the cosmic rays produced by the formation of the heavier of the common elements like silicon and iron out of hydrogen, the observed absorption coefficients will be somewhat smaller than those computed from the energy available for their formation. This is precisely the behaviour which our cosmic ray depth-ionisation curve actually reveals. At the highest altitudes at which we have recently observed $(14,000 \mathrm{ft}$.), the helium rays have reached equilibrium with their secondaries, and the observed and computed coefficients agree as they should. For the oxygen rays the observed coefficent is a little lower than the computed value -about 17 per cent lower; for the silicon rays still lower-about 30 per cent; and for the iron rays considerably lower still-about 60 per cent : all in beautiful qualitative agreement with the theoretical demands as outlined.

The foregoing results seem to point with much definiteness to the following conclusions:

1. The cosmic rays have their origin not in the stars but rather in interstellar space.

2. They are due to the building up in the depths of space of the commoner heavy elements out of hydrogen, which the spectroscopy of the heavens shows to be widely distributed through space. That helium and the common elements oxygen, nitrogen, carbon, and even sulphur, are also found between the stars is proved by Bowen's beautiful recent discovery that the 'nebulium lines' arise from these very elements.

3. These atom-building processes cannot take place under the conditions of temperature and pressure existing in the sun and stars, the heats of these bodies having to be maintained presumably. by the atom-annihilating process postulated by Jeans and Eddington as taking place there.

4. All this says nothing at all about the second law of thermodynamics or the Wärme-Tod, but it does contain a bare suggestion that if atom formation out of hydrogen is taking place all through space, as it seems to be doing, it may be that the hydrogen is somehow being replenished there, too, from the only form of energy that we know to be all the time leaking out from the stars to interstellar space, namely, radiant energy. This has been speculatively suggested many times before, in order to allow the Creator to be continually on his job. Here is, perhaps, a little bit of experimental fingerpointing in that direction. But it is not at all proved or even perhaps necessarily suggested. If Sir James Jeans prefers to hold one view and I another on this question, no one can say us nay. The one thing of which we may all be quite sure is that neither of us knows anything about it. But for the continuous building up of the common elements out of hydrogen in the depths of interstellar space the cosmic rays furnish excellent experimental evidence. I am not unaware of the difficulties of finding an altogether satisfactory kinetic picture of how these events take place, but acceptable and demonstrable facts do not, in this twentieth century, seem to be disposed to wait on suitable mechanical pictures. Indeed, has not modern physics thrown the purely mechanistic view of the universe root and branch out of its house?

\section{Geodesy in India.}

$I^{N}$ the British Empire at the present time, geodetic operations are mainly confined to Canada, India, and South Africa. The Dominion and the Union are working principally for the more pressing needs of development; in India, on the other hand, apart from the necessity for revision, more attention is being paid to the interpretation of results. The Great Trigonometrical Survey of India itself being long complete, triangulation is now being carried on in the outer zones-in Burma and on the Siamese frontier at the date of the last Geodetic Report. ${ }^{1}$

The main triangulation in 1928-29 was executed with Wild theodolites, which gave very good results when the instruments were working. Their axes, however, stiffened in the field, causing serious loss of time. Surveyors cannot adjust the instruments in the field, and even the mathematical instrument workshop in Calcutta found adjustment difficult, though mere oiling is simple if the method is known. It was intended to keep the older and heavier 12-inch theodolites at hand during the ensuing season, in case of further failures.

Precise levelling is perhaps the most economic- ally important section of the revisionary geodetic work: of the new net of 16,000 miles proposed, nearly one-half was completed in 1929. Levelling on hilly circuits appears to show that the shorter sights thereon contribute to accuracy as against longer sights in flat country; experience in precise levelling has given revised results on hilly circuits in Ceylon which are practically as good as on the plains. Indian investigations show that error due to differential refraction on steep slopes is negligible, and the greater part of the errors of closure is believed to be due to changing length of the staves. The results of levelling must lie within limits of accidental and systematic error which are strictly defined; one notices that 55 per cent of one line was relevelled. On the several lines-not yet, of course, referred strictly to M.S.L.- the relative discrepancies between the new and the old measures do not ordinarily exceed 6 inches; but there are interesting exceptions. Thus there is evidence of a sinkage around Ambala of about an inch per decade, attributed to removal of water from wells. On the line between Sukkur and Hyderabad the results of much levelling have given 